OPEN ACCESS

Edited by:

Paul J. Colombo,

Tulane University, United States

Reviewed by: Fabiana Silva Ribeiro, Portuguese Catholic University, Portugal Lutz Jäncke,

University of Zurich, Switzerland

*Correspondence: Katri Annukka Saarikivi katri.saarikivi@helsinki.fi

Received: 17 June 2019 Accepted: 07 October 2019 Published: 06 November 2019

Citation:

Saarikivi KA, Huotilainen M, Tervaniemi $M$ and Putkinen $V$ (2019) Selectively Enhanced Development of Working Memory in Musically Trained Children and Adolescents. Front. Integr. Neurosci. 13:62. doi: 10.3389/fnint.2019.00062

\section{Selectively Enhanced Development of Working Memory in Musically Trained Children and Adolescents}

\author{
Katri Annukka Saarikivi ${ }^{1,2 *}$, Minna Huotilainen ${ }^{2,3}$, Mari Tervaniemi ${ }^{2,4}$ and Vesa Putkinen ${ }^{2,5}$ \\ ${ }^{1}$ Cognitive Brain Research Unit, University of Helsinki, Helsinki, Finland, ${ }^{2}$ Department of Psychology and Logopedics, \\ Medicum, Faculty of Medicine, University of Helsinki, Helsinki, Finland, ${ }^{3}$ Faculty of Educational Sciences, University of \\ Helsinki, Helsinki, Finland, ${ }^{4} \mathrm{CI}$ CERO Learning, Faculty of Educational Sciences, University of Helsinki, Helsinki, Finland, \\ ${ }^{5}$ Turku PET Centre, Turku, Finland
}

In the current longitudinal study, we investigated the development of working memory in musically trained and nontrained children and adolescents, aged 9-20. We measured working memory with the Digit Span (DS) forwards and backwards tests $(N=106)$ and the Trail-Making A and B (TMT-A and B; $N=104$ ) tests three times, in 2011, 2013, and 2016. We expected that musically trained participants would outperform peers with no musical training. Indeed, we found that the younger musically trained participants, in particular, outperformed their nontrained peers in the TMT-A, TMT-B and DS forwards tests. These tests all primarily require active maintenance of a rule in memory or immediate recall. In contrast, we found no group differences in the backwards test that requires manipulation and updating of information in working memory. These results suggest that musical training is more strongly associated with heightened working memory capacity and maintenance than enhanced working memory updating, especially in late childhood and early adolescence.

Keywords: musical training, longitudinal, working memory, updating, maintenance, development, trail-making test, Digit Span

\section{INTRODUCTION}

Musically trained individuals have been reported to outperform musically nontrained peers in various kinds of cognitive tests not directly related to music-making, including ones measuring long-term verbal and visual memory (Chan et al., 1998; Ho et al., 2003), executive functions (Bialystok and Depape, 2009; Degé et al., 2011; Moreno et al., 2011; Zuk et al., 2014; Saarikivi et al., 2016; however, see Schellenberg, 2011), and even intelligence (Schellenberg, 2004, 2006; Moreno et al., 2011). Executive functions (Stuss and Alexander, 2000; Jurado and Rosselli, 2007; Diamond, 2013) are cognitive processes typically divided into three related components: working memory, inhibition, and cognitive flexibility (Miyake et al., 2000; Lehto et al., 2003; Miyake and Friedman, 2012; Diamond, 2013). These three processes allow individuals to acquire, maintain, manipulate, and update representations of information of the environment, and monitor, direct and alter behavior according to these representations. Multicomponent models of working memory propose subprocesses for maintaining representations of information in memory and for manipulating this information. For instance, the influential 
model of Baddeley and Hitch (1974) divided working memory into two components for storage and manipulation of verbal and visual material, and a cognitive control unit (for other models, see e.g., Cowan, 1988, 1999; Unsworth and Engle, 2007). Neuroimaging and lesion studies have found separate neural functions for memory representations and attention processes that govern manipulation of this information, supporting these modular views of working memory (Postle et al., 1999; Gerton et al., 2004; Owen et al., 2005; reviews: D'Esposito et al., 1995; Miller and Cohen, 2001; Linden, 2007; Nee et al., 2012; Rottschy et al., 2012; Eriksson et al., 2015; for a discussion on differences between short-term memory and working memory, see e.g., Unsworth and Engle, 2007; Cowan, 2008; Aben et al., 2012). Multicomponent models of working memory have been validated in child studies (Gathercole et al., 2004; Gray et al., 2017), and separate brain mechanisms for encoding, maintenance and retrieval of verbal information have also been found in neuroimaging studies of children, from the age 6 onwards, and in adolescents (Gathercole et al., 2004; Siffredi et al., 2017).

Working memory and other executive functions develop from early childhood until adolescence (Cepeda et al., 2001; De Luca et al., 2003; Vuontela et al., 2003; Luna et al., 2004; Zelazo et al., 2004; Huizinga et al., 2006), following the maturation of prefrontal areas (Casey et al., 2000; Fuster, 2002; Kwon et al., 2002; Bunge and Wright, 2007; Kharitonova et al., 2013). Different executive functions however mature at slightly different rates. Development of shifting ability, which is related to cognitive flexibility, has been found to continue until adolescence (Huizinga et al., 2006; Best and Miller, 2010; Huizinga and van der Molen, 2011), and development of working memory even further, until early adulthood (Kwon et al., 2002; Huizinga et al., 2006; Satterthwaite et al., 2013).

Several cross-sectional studies have reported varying musician advantage in working memory tasks. For example, in a study by George and Coch (2011), years of musical training correlated positively with scores in both verbal and visual span tests for memory in college-aged individuals. Similarly, in another study (Talamini et al., 2016), musically trained adults outperformed nontrained peers in auditory as well as visual span tests for working memory. Finally, Zuk et al. (2014) found better performance in the Digit Span backwards test in adult musicians compared to nonmusicians, but not in musically trained children compared to nontrained peers.

Longitudinal studies with children suggest that the putative musician advantage in memory tasks may be caused by training and does not solely reflect pre-existing differences (for a discussion on problems of inferring causation from these kinds of studies, see Schellenberg, 2015). In the study by Ho et al. (2003), verbal long-term memory improved in children who continued musical training during a year-long follow-up, but not in those who did not. Similarly, in a study following the development of musically trained and nontrained children (Bergman Nutley et al., 2014), musical training was associated with improvement of verbal working memory as measured by the backwards Digit Span test, but also visual working memory as measured by a visuo-spatial working memory task.
Another longitudinal study (Fujioka et al., 2006), comparing the development of children who undertook music lessons for 1 year to the development of children in a Control group, found significant improvement of working memory as measured by the Digit Span test only in the Music group. In another study (Roden et al., 2014), improvement of working memory was observed in preschool-aged children after 18 months of musical training, but not in an active Control group. In the study, effects were found specifically in tests measuring the phonological loop and the central executive subcomponents of working memory. The phonological loop was measured with the One Syllable Word Span Test, requiring participants to memorize and recite a sequence of words in the order they were presented, and the Nonword recall test, requiring participants to recite a nonword immediately after hearing it. The central executive was measured by complex span tasks requiring processing and storing information at the same time or requiring reversal of the order of a memorized sequence of information units. Last, in a recent study with quasi-random assignment of children into musical training and Control groups (Guo et al., 2018), it was found that 6 weeks of musical training improved working memory. Auditory working memory was assessed with the Digit Span forward and backward tests and with the Letter-Number Sequencing test. Both require working memory maintenance of aurally acquired information and updating and manipulation of that information in memory.

The notion that musical training might influence memory skills is further supported by findings of training-related changes in brain structures important for working memory. In their seminal study on structural differences, Gaser and Schlaug (2003) found that musicians had greater gray matter density in areas important for motor and auditory processes, and also a region of the cerebellum connected to working memory (Stoodley et al., 2012). Similarly, in a study by James et al. (2014), musical training correlated positively with gray matter density in a cerebellar areas and basal ganglia important for working memory. Another MRI study found increased thickness of frontal areas related to working memory in musicians, when compared to non-musicians (Bermudez et al., 2008).

Musical training has also been connected to changes in brain functions related to working memory. In the study by George and Coch (2011), musicians had shorter latencies of electrical brain responses (P3) to changes in visual as well as auditory stimuli, as well as larger P3 amplitudes to tonal changes. The P3 response is thought to reflect updating of working memory. In a recent study (Cheung et al., 2017), musically trained individuals outperformed nontrained peers in tasks for verbal memory and also differed in electrical brain activity measured during a verbal memory task. Specifically, musically trained individuals showed more intrahemispheric coherence in the theta band. In an fMRI study (Pallesen et al., 2010), musicians showed greater activation of brain networks for attention and working memory, including frontal, parietal, and subcortical areas than nonmusicians. In another study (Schulze et al., 2011), musicians showed different patterns of activation of brain areas during memory encoding and rehearsal of structured and unstructured tonal sequences. Nonmusicians did not show differences in activation patterns 
during these tasks. Musicians also outperformed nonmusicians in learning the tonal sequences.

A recent meta-analysis on cross-sectional and longitudinal studies on music-related enhancement of working memory in children and adults (Talamini et al., 2017) concluded that musicians and musically trained individuals have a clear advantage across different memory tasks when compared to nontrained peers. However, according to the results, the effect size depended on the type of information that was processed and on the memory processes required by the task. In general, the musician advantage was stronger for working memory than long-term memory, and for auditory rather than visual stimuli.

Another recent meta-analysis (Sala and Gobet, 2017a) found only a weak musician advantage in memory tasks. In this metaanalysis, however, no distinction was made between working memory and long-term memory, which may have obscured the effects of musical training on working memory reported in several cross-sectional and longitudinal studies.

In sum, there is evidence of an association between musical training and specifically verbal working memory. However, longitudinal studies have focused on school- or preschool-aged children even though executive functions are known to develop long into adolescence. As a result, it is still unclear how musical training specifically augments the development of working memory, and for how long into adulthood the possible advantage persists.

In this longitudinal study, we compared the working memory skills of 114 musically trained and nontrained children and adolescents aged 9-20. During this age range, executive functions including working memory undergo significant development, owing to the protracted development of brain areas such as the frontal lobes that important for these skills, but also begin to reach maturity (Taylor et al., 2013, 2015) The sample allows for investigating the effects of musical training on working memory, and the persistence of these effects during a developmentally highly interesting window of time. The study aims at answering questions that remain unresolved in research examining the effects musical training may have on cognitive development: does musical training augment the development of working memory, does musical training produce an advantage in working memory tasks, does this advantage persist into adulthood?

To investigate working memory, we employed two broadly studied and well-established tests: the Digit Span backward and forwards tests and the Trail-Making Test A and B. Data on performance in Digit Span tests were collected during a 3-year follow-up and the TMT-A and B tests during a 2 year followup. Based on previous literature, we expected musically trained children and adolescents to perform better than nontrained peers in all tests.

\section{MATERIALS AND METHODS}

\section{Participants}

Altogether 106 children and adolescents aged 9-20 years participated in the study (Tables 1,2 ). The musically trained participants $(N=54,32$ females $)$ had started training on a musical instrument approximately at age 7 . They had attended or were currently attending a public elementary school that emphasizes music in the curriculum. In addition to weekly classes in classical instrumental training, school days contained music lessons such as choir and ensemble training and performances. Thus, at age 9, participants had a total of approximately 2 years of musical training and participation in the musical curriculum, at age 11,4 years and so on. The nontrained participants $(N=52$, 26 females) had no formal training on a musical instrument. They attended or had attended a standard elementary school with weekly group-based music lessons until the age of 13, but no instrumental tuition. No children reported hearing deficits or neurological impairments. The Music and Control groups were matched in SES and IQ (Putkinen et al., 2014).

Written informed consent for participation was obtained from guardians of underaged participants or from over 18-year old participants themselves before the experiment. All participants also gave verbal consent for their participation. Participants were rewarded three movie tickets for taking part in the measurement. The experiment protocol was approved by the Ethical Committees of the Department of Psychology and of the Faculty of Behavioural Sciences, both at the University of Helsinki, Finland.

\section{Working Memory Tests}

The Digit Span forwards and backwards (DS forwards, DS backwards) tests (WISC-IV, Wechsler, 2010) as well as the TMT-A and B (TMT-A, TMT-B; Poutiainen et al., 2010) were used to measure verbal working memory. In the Digit Span forwards test, subjects are aurally presented with a series of digits, and immediately recite them from memory. In the DS backwards test, participants are required to recite the presented digits in reverse order. There are multiple presentation rounds, with the experimenter always adding one to-be-memorized digit. The forwards test requires active maintenance of information in mind, and the backwards tests also manipulation of this information. Performance is evaluated by the total of digits that the participant is able to correctly recite.

The TMT-A requires the participant to connect digits printed randomly on paper by drawing a line from number to number in a sequential order. The Trail-Making Test B requires participants to alternate between connecting numbers and letters printed on the paper in order (1-A-2-B-3-C...). Both tests require maintenance of the rule of the task in mind and also maintaining awareness of where one is progressing on the sequence of digits (A) and both digits and letters (B). Performance is measured by the time taken to complete the test.

\section{Procedure}

This study is part of a longitudinal study that started in 2003 investigating the maturation of auditory processes and executive functions in children undergoing musical training and a control group. The study entailed also EEG measurements and other tests for various cognitive skills (these data are reported elsewhere). Measurements were conducted every 2 years, with a new group of 7-year-olds recruited every 2 years. The data, therefore, contains measurements from the same participants 
but from different years. The data reported here include measurements conducted in the years 2011, 2013, and 2016 for the DS forwards and backwards tests and from the years 2011 and 2013 for the Trail-Making A and B Tests (The TMT was not conducted in 2016). Not all children took part in every measurement. For the tests, 25 children participated in only one measurement, 43 in two, and 38 in all three measurements. For the Trail-Making A and B Tests, 43 children participated in one measurement and 61 in both.

The cognitive tests were conducted before the EEG experiments and took a maximum of $1 \mathrm{~h}$ altogether. Upon arrival at the laboratory, written informed consent as well as oral consent was received from the participants. After this, the participants accompanied the experimenter to a room to complete the tests. Experimenters were graduate students, trained to work with children and adolescents and to administer the tests. The space was a comfortably lit sound-proofed room, previously used as an EEG lab, converted for testing use. The experimenter and the subject were orthogonally seated at a table. After the tests were completed, the subject was escorted to the EEG lab, where the EEG cap was attached, and the subject informed more closely about the EEG experiment. EEG measurement ensued. Participants were offered bathroom breaks when needed, and cookies and juice before the EEG measurement as well as half way through it.

\section{Statistical Analysis}

Completion times in the TMT-A and B, and span (number of correctly recited digits) in DS forwards and backwards were included separately in analyses of test performance. The effect of age and group membership on test performance was modeled with linear mixed modeling using the lmer function [Test Score $\sim$ Age * Group + (1|Subject)] of the Lme4 package in R (Bates, 2005; Bates et al., 2007). Age was mean centered so that the significant effect of Group indicates a group difference in the test score at average age of the participants (mean ages for the DS and TMTs were 14.39 and 13.44 years, respectively). Linear mixed modeling was selected as the analysis approach since it allows a different number of data points across subjects and takes into account the correlated nature of the data within a subject. Values below the Q1-1.5* IQR (inter-quantile-range) or above Q3 + 1.5* IQR were classified as outlier and replaced by the lower or upper cutoff values of this range, respectively. This procedure was applied twice for the DS backwards and Trail Making A data and five times for the Trail Making B data.

\section{RESULTS}

Performance of participants in all tests except for the DS backwards test improved with age (Figures 1, 2). Musically trained participants outperformed nontrained participants in the DS forwards test, but not in the backwards test. The musically trained individuals also outperformed nontrained peers in the TMT-A. However, the group difference depended on age. The difference between performance in the Music and Control groups decreased with age. A similar age-dependent effect was also found for performance in Trail-Making Test B. The results are described in more detail below.

\section{Digit Span Forwards and Backwards}

Performance in the DS forwards test improved with age (estimated increase in span per year: $0.22, p<0.001$ ). The Music group outperformed the Control group (estimates for the Music and Control groups: Control difference in span: 0.56, $p<0.05$ ). The model revealed no evidence that this group difference was dependent on age (Group*Age interaction, $n s$ ).

For the DS backwards test, there were no significant effects of Age or Group or and no significant interaction between these predictors.

\section{Trail-Making A and B Tests}

Subjects' performance in the TMT-A improved with age (estimate for the decrease in completion time per year: -2.87 , $p<0.001)$. There was a trend-level effect of group suggesting that the Music group outperformed the Control group in this test (estimate for the Music < Control difference: $-2.50, p<0.07$ ). However, there was also a significant interaction between Age and Group indicating that the group difference was more pronounced in the younger children and decreased with age (estimate for the Music < Control differences in the change in completion time per year: $1.11, p<0.05)$.

The performance in the Trail-Making Test B also improved with age (Estimate for the decrease in completion time per year: $-9.25, p<0.001)$. For this test, there was a significant effect of group indicating that the Music group outperformed the Control group (estimate for the Music < Control difference: -12.06 , $p<0.05)$ as well as a significant interaction between Age and Group indicating that this group difference decreased with age (estimate for the Music < Control differences in the change in completion time per year: $4.69, p<0.05$ ).

\section{DISCUSSION}

In this study, we investigated the development of working memory in musically trained and nontrained children and adolescents. Musically trained participants outperformed nontrained peers in the DS forwards test as well as the TrailMaking A and B tests. Furthermore, the group difference in the two latter tests decreased with age. We did not find a significant difference between the Music and the Control groups in the DS backwards test.

\section{A Musician Advantage in the DS Forwards and Trail-Making $A$ and $B$ Tests}

The better performance of the Music group in the DS forwards test concurs with previous research showing a musician advantage in tests for memory (Chan et al., 1998; Fujioka et al., 2006; George and Coch, 2011; Bergman Nutley et al., 2014; Roden et al., 2014; Zuk et al., 2014; Talamini et al., 2016; Guo et al., 2018; review: Talamini et al., 2017). It is noteworthy, however, that studies reporting memory enhancement in musicians have conceptual and methodological differences. In the study by Cheung et al. (2017), verbal memory enhancement was reported 

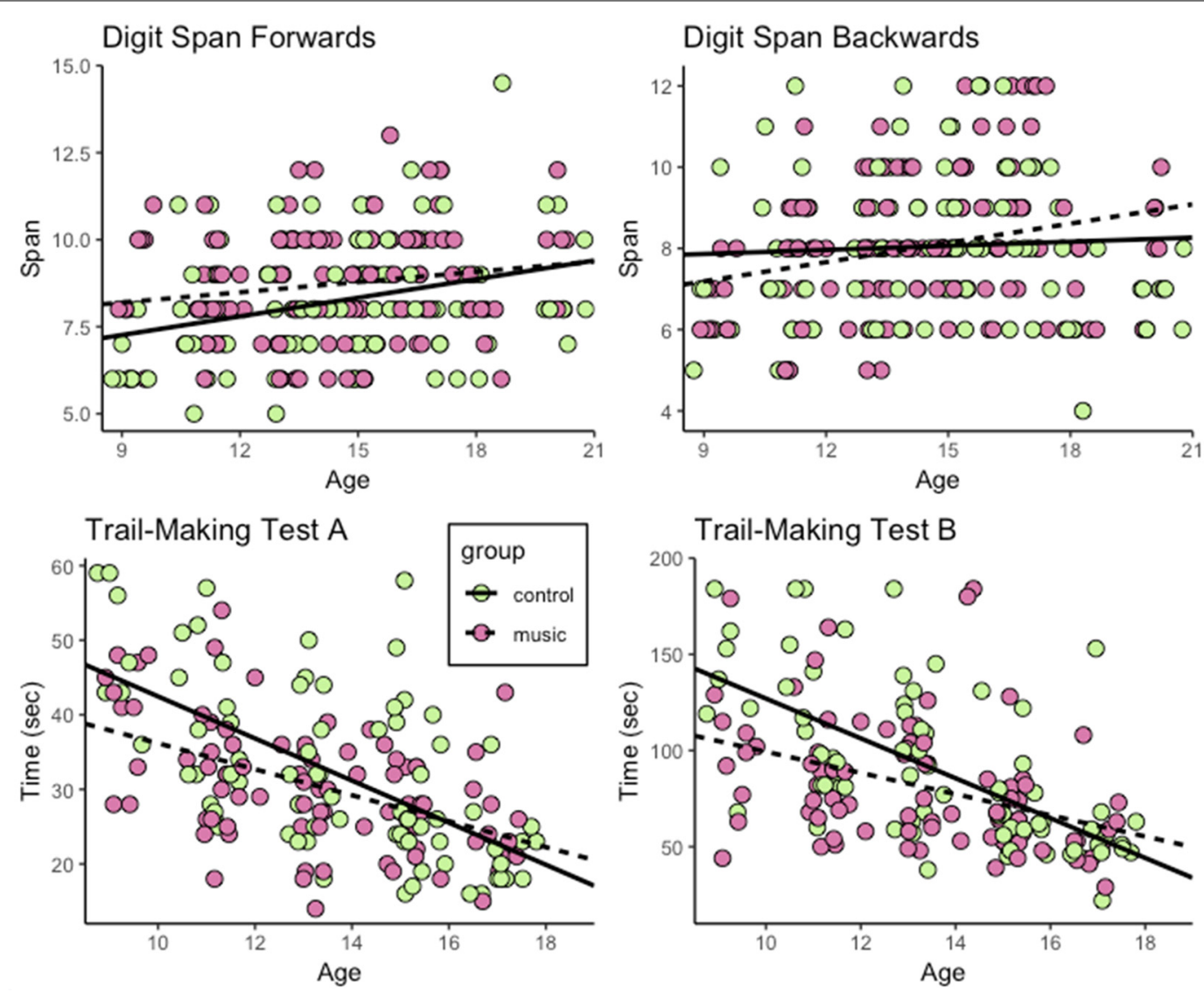

FIGURE 1 | Performance of participants in the Digit Span forwards and backwards, and the Trail-Making A and B tests across all age groups. Music and Control groups represented with different colors.

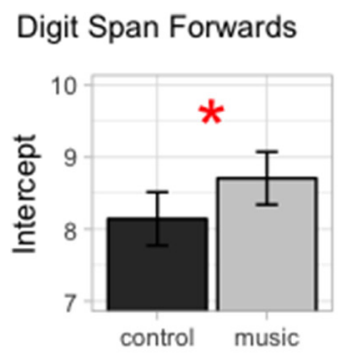

Trail-Making A

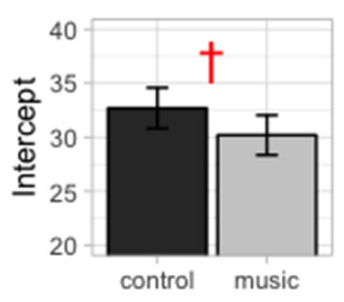

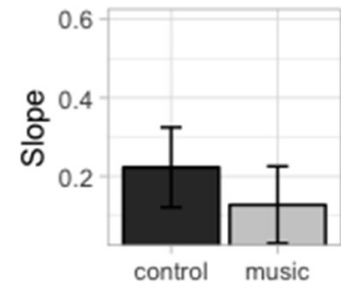

control music
Digit Span Backwards
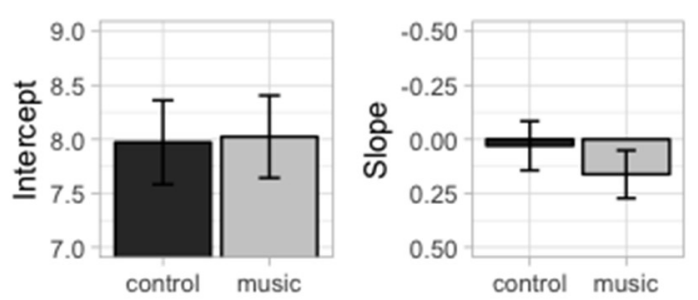

Trail-Making B
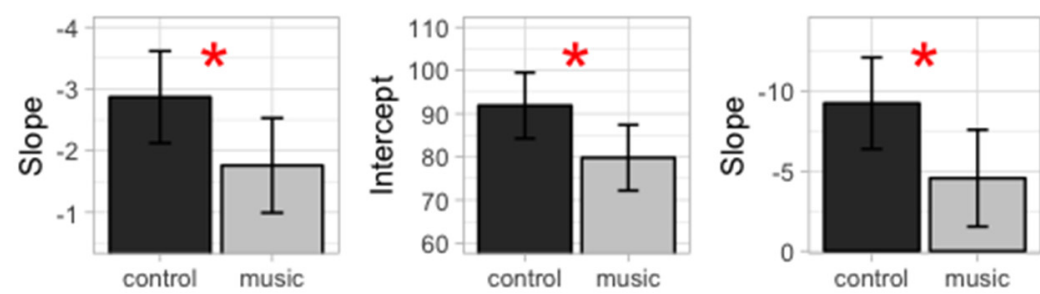

FIGURE 2 | The intercepts (i.e., estimated performance at mean age) and slopes (i.e., estimated change in performance with age) for each test separately for the Music and Control groups. The errorbars indicate $95 \%$ confidence intervals. ${ }^{*} p<0.05,{ }^{\dagger} p=0.07$. 
TABLE 1 | Ages of participants in the Music and Control groups per measurement year for the Digit Span Test.

\begin{tabular}{|c|c|c|c|c|c|}
\hline Group & Year & Mean age & Minimum age & Maximum age & Standard deviation \\
\hline Control & 2011 & 12.77 & 8.75 & 15.92 & 2.26 \\
\hline Control & 2013 & 14.61 & 10.43 & 17.81 & 2.45 \\
\hline Control & 2016 & 17.25 & 13.56 & 20.78 & 2.51 \\
\hline Music & 2011 & 12.46 & 8.92 & 15.83 & 2.31 \\
\hline Music & 2013 & 14.09 & 10.60 & 17.44 & 2.20 \\
\hline Music & 2016 & 16.76 & 13.50 & 20.29 & 2.30 \\
\hline
\end{tabular}

TABLE 2 | Ages of participants in the Music and Control groups per measurement year for the Trail-Making Test.

\begin{tabular}{|c|c|c|c|c|c|}
\hline Group & Year & Mean age & Minimum age & Maximum age & Standard deviation \\
\hline Control & 2011 & 12.77 & 8.75 & 15.92 & 2.26 \\
\hline Control & 2013 & 14.61 & 10.43 & 17.81 & 2.45 \\
\hline Music & 2011 & 12.46 & 8.92 & 15.83 & 2.31 \\
\hline Music & 2013 & 14.09 & 10.60 & 17.44 & 2.20 \\
\hline
\end{tabular}

based on performance in a task requiring immediate as well as delayed recall of a word list, i.e., working memory as well as long-term memory. In contrast, the current study focused on working memory and employed the classical DS measure. Therefore, this study adds to the evidence for enhanced working memory in musically trained children along with earlier longitudinal studies that have used the similar span tests (Fujioka et al., 2006; Bergman Nutley et al., 2014; Guo et al., 2018).

Interestingly, we found enhancement of performance in only the forwards and not the DS backwards test. Similar results have been obtained in the study by Hansen et al. (2013) who found that musical training was associated with better performance in the DS forwards, but not backwards test. Furthermore, in their study, DS forward performance was connected to performance in musical ability tests. Along the same lines, Lee et al. (2007) found that musically trained adults outperformed nontrained peers in forwards, but not DS backwards. In their study, musically trained children, aged 12 on average, however, outperformed nontrained peer both in the forwards and DS backwards tests. Guo et al. (2018), in turn, found enhancement of the backwards but not the DS forwards after a short-term instrumental training program. Likewise, Bergman Nutley et al. (2014) found only enhancement of DS backwards performance in musically trained adults and children, but unfortunately they did not include DS forwards to allow for comparison. Thus, the literature is mixed as to whether musical training is associated with enhancement of forwards or DS backwards or both.

In any case, the current study found longitudinal evidence in a large sample of subjects in favor of selective enhancement of DS forwards in musically trained children and adolescents. Although negative results cannot be taken as evidence for the null hypothesis that there is no difference between the groups in DS backwards, the substantial statistical power of the current study indicates that a putative undetected group difference in DS backwards would have to be very small and of little practical importance.

In this study, we also found that musically trained participants outperformed nontrained peers in both of the TrailMaking Tests. Previously, adult musicians have been found to outperform nonmusicians in TMT-A and B (Bugos and Mostafa, 2011), or TMT B alone (Strong and Mast, 2019). However, for instance Bialystok and Depape (2009) and Virtala et al. (2014) found no differences between adult musicians and nonmusicians in span tests or the TMT-A or B. Our results concur with previous findings of enhanced performance in TMT-A and B in musically trained individuals but extend these findings to children and adolescents.

\section{Working Memory Subcomponents Measured by the Digit Span Test and the Trail-Making Test}

The Digit Span test has usually been categorized as a simple span test, requiring maintenance of information in memory. Complex span tests in turn require memory maintenance of information during another, unrelated cognitive operation (Wilhelm et al., 2013). However, in a meta-analysis conducted by Redick and Lindsey (2013), the correlation between DS backward performance and performance in n-back tasks as well as a verbal complex span tests was greater than the correlation between DS forward and these tests. Because the DS backward test requires subjects to reverse the order of the strings presented in mind, it also requires working memory updating. Furthermore, the DS forward and backward tests have both been found to recruit in part separate brain networks (Manan et al., 2014). Both activate areas connected to working memory, but with the backward test more strongly activating brain areas related to cognitive control and phonological processing (Gerton et al., 2004; Yang et al., 2015).

As the DS forwards and backwards tests have been found to recruit in part separate working memory processes, it is possible that performance in one but not the other could be enhanced through training. Indeed, selective enhancement of working memory updating (Linares et al., 2018, 2019) and maintenance (Carretti et al., 2007) skills has been found as a result of working memory training in adults. Another study achieved selective impairment of working memory maintenance, but not updating with tDCS (Wang et al., 2018). Our findings of musician advantage in DS forwards, but not backwards points 
towards selective enhancement of working memory maintenance but not updating.

The Trail-Making Test is usually used to measure executive functions, and neuroimaging and lesion studies have identified that TMT recruits large-scale fronto-parietal brain networks related to these functions (Varjacic et al., 2018). However, there is evidence that performance in the TMT is related primarily to processing speed and working memory ability, as well as fluid intelligence (Sánchez-Cubillo et al., 2009; Satterthwaite et al., 2013). These findings are supported by evidence of genetic correlations between trail-making performance, reasoning ability and general cognitive ability, processing speed, and memory (Hagenaars et al., 2018). Research has also found differences between the cognitive processes underlying TMT-A and B performance. TMT-A is thought to rely mainly upon processing speed, and TMT-B to additionally require working memory and switching ability (Arbuthnott and Frank, 2000; Sánchez-Cubillo et al., 2009). According to a validation study of a computerized version of the TMT, TMT-B performance was explained to a large degree by inhibition and visual working memory skills (Fellows et al., 2017). Similar results were obtained in a factor analysis of TMT performance and several other neurocognitive measures in older individuals, where TMT-B performance was connected to measures of working memory and inhibition, and TMT-A to processing speed (Llinàs-Reglà et al., 2017).

There is also significant overlap between the cognitive processes that the TMT recruits. For instance, working memory skills and working memory capacity are tightly related to fluid intelligence (Kane et al., 2005; Kail, 2007; Demetriou et al., 2014; Salthouse, 2014; Heinzel et al., 2016). It has also been found that working memory predicts switching (Blackwell et al., 2009), presumably through supporting the maintenance of switching rules. Inhibitory control, in turn, may have a role in supporting working memory maintenance (Jonides et al., 1998; Zanto and Gazzaley, 2009; Getzmann et al., 2018).

In behavioral studies, DS backwards performance has been found to predict TMT-B performance, suggesting a partial overlap between the cognitive requirements of these tasks (Sánchez-Cubillo et al., 2009). Both DS backwards and TMT-B engage cognitive control more than DS forwards and TMT-A, but there are also obvious differences between test requirements. TMT-B requires switching attention from one rule and sequence of information in memory to another (letters or numbers). It also requires continuous updating of information about the respondent's position along the series of letters or numbers they are connecting. DS backwards requires recoding a string of digits in mind into reverse order, or updating the representation of the acquired information, but does not require switching between rules or response patterns during responding. The TMT-A and $\mathrm{B}$ also engage specifically working memory maintenance, by requiring the participant to keep the response rule and progression along the sequence of letters and numbers in mind. As in this study, we observed a musician advantage in DS forwards but not backwards, and both the TMT-A and $B$, our results point towards enhancement of skills that are required by these tests but not by the DS backwards test. These include working memory maintenance for DS forwards, as well as TMT-A and B. TMT-B also requires switching ability, not required by the DS backwards test. In addition to working memory maintenance, the musician advantage in TMT-B can therefore also be explained by enhancement of switching ability.

In sum, while the Digit Span and the Trail-Making Test are routinely used to assess and connected to working memory ability, the task impurity problem complicates reaching conclusions about specifically which cognitive functions are measured and to what extent. Our results are best explained by enhancement of working memory maintenance, required by the TMT-A, B and the DS forward test. In addition, enhancement of switching ability may explain the musician advantage in TMT-B.

\section{How Musical Training Could Exert Selective Effects on the Development of Working Memory}

Learning to play a musical instrument or sing requires working memory in a multitude of ways. For example, memorizing and producing sequences of tones when learning music by heart, and responding to changes in music when playing together with others both require working memory. It is possible that musical training during childhood could enhance working memory to the extent that this could be seen as faster development of these skills.

Augmentation of memory skills has been obtained by working memory programs (Melby-Lervåg and Hulme, 2013; Sala and Gobet, 2017b). It has been suggested that programs focusing on core working memory skills are most effective (Morrison and Chein, 2011). These programs are characterized by tasks that contain stimuli in more than one modality, require working memory maintenance and interference control, quick memory encoding and retrieval, change according to the individual's skill level and require high engagement and focus (Morrison and Chein, 2011). Musical training matches these characteristics of core working memory training programs well. For instance, learning to play sheet music requires transformation of visual stimuli into motor actions, which produce sound stimuli. Playing from notes requires concurrent working memory maintenance and updating of visual information from notation and auditory information produced by the musician. Playing from memory adds to working memory updating and maintenance demands through requiring monitoring of the sounds and movements produced and matching them to the model of the musical piece in memory. In ensemble playing, interference control is needed in order to be able to segregate the stream of sound produced by the individuals from those produced by others. Ensemble playing also requires rapid working memory encoding and retrieval, as musicians need to follow not only their own stream of sound but also that of others, and respond to changes in others' output. In joint improvisation, these rapid working memory encoding and retrieval requirements are accentuated. Musical training increases in challenge according to the proficiency of the individual, and successful learning and playing of music requires great engagement and focus. It is 
therefore feasible that musical training might influence working memory processes.

The results on selective enhancement of the participants' working memory maintenance, but not working memory updating skills, would mean that musical training selectively engages these mechanisms and perhaps selectively supports development of one more than the other. This explanation resonates with findings of different patterns of brain activation during memory encoding and rehearsal, reflecting differences in memory processes in musicians compared to nonmusicians (Schulze et al., 2011). It is feasible that musical training might exert powerful effects specifically on working memory maintenance. Learning to play by ear relies heavily on an individual's capability of acquiring and storing auditory information, melodies, and then reproducing this information immediately. Learning to play from notes, in turn, hones working memory maintenance in the visual domain. Conversely, classical musical training may not as much emphasize the ability to augment the presented information in mind, but rather reproduce it exactly as presented.

An alternative explanation for selective enhancement of working memory maintenance is that musical training improves selective attention. Indeed, there is evidence that selective attention underlies working memory maintenance (Sreenivasan and Jha, 2007; Berry et al., 2009; Gazzaley and Nobre, 2012). Selective attention seems to support encoding and maintenance of information in memory by shielding it from distracting information. This notion is supported by neuroimaging evidence of attenuated processing of distracting information during a working memory maintenance task (Sreenivasan and Jha, 2007). There is also tentative evidence of a musician advantage selective attention, indexed by decreased variability of frontal brain responses to attended stimuli (Strait and Kraus, 2011; Strait et al., 2015). It is possible that music training, for instance through playing in ensembles, and learning to focus on only the sound produced by one's instrument, could develop selective attention, which is of benefit in tasks requiring memorization of aurally presented information. Selective attention may also be required and therefore trained in learning to play sheet music. For instance, in learning to play piano, there are two notations to follow-one for the right and one for the left hand. Selectively attending to this visual information is required for successful production of sound. Trail-Making Test performance has been connected to selective attention skills, as measured by the ability to recognize speech in noise (Ellis et al., 2016), but to the knowledge of the authors, similar results on a connection between specifically selective attention and Digit Span performance have not been obtained. In future studies investigating working memory in musically trained individuals, including measures for selective attention would help further elucidate this possible connection.

\section{Augmented Developmental Trajectories in Trained and Nontrained Participants}

The difference in performance between the Music and Control group observed in this study diminished over time. It is possible that musical training enhances the development of working memory maintenance or selective attention, which can be seen as faster maturation in the Music group, but with time the Control group children attain the same level of performance. This explanation is contrasted by studies that have found enhanced working memory still in musically trained adults (Chan et al., 1998; Bialystok and Depape, 2009; George and Coch, 2011; Zuk et al., 2014; Talamini et al., 2016; Ding et al., 2018). There are, however, also contrary findings. In one study, adult nonmusicians were found to outperform musicians in tests requiring immediate as well as delayed recall of newly acquired information, with no significant group differences in performance in the TMT-A or B or DS (Virtala et al., 2014). Thus, the existence of working memory benefits associated with musical expertise in adulthood should be considered with caution.

As stated before, the task impurity problem complicates understanding of which cognitive functions are putatively most affected by musical training. Furthermore, the maturation of other executive functions may influence the maturation of subprocesses of working memory. For instance, the protracted development of inhibitory control and shifting ability influence performance in complex working memory span tasks that require these skills in addition to maintaining information in working memory (Jonides et al., 1998; Schleepen and Jonkman, 2009). Future longitudinal studies investigating working memory development should include measures that allow for disentangling the unique contributions of development in these cognitive skills to the development of working memory.

Since our study lacks baseline measurement of working memory skills prior to musical training, our results may also be explained by pre-existing differences between the two groups, instead of developmental causal explanation (for a study pointing towards pre-existing differences in intelligence, which may explain better performance in executive functions, see Schellenberg, 2015). The lack of the baseline measurement is caused by our choice to minimize the length of the experimental session when the children were only 7 years old and about to start their instrumental training. We added more behavioral and ERP paradigms gradually when the children became older and could then better cope with longer sessions. By this arrangement, we were able to minimize the number of drop-out participants-a serious problem in all longitudinal studies (for discussion, see Tervaniemi et al., 2018; Barbaroux et al., 2019).

One might consider the lack of random group allocation also as a caveat of our study. However, in our view, it is not feasible to plan a longitudinal study for several years on children and adolescents, at least if a control group is included. If the participants are not motivated, they either quit the training, do not participate in the investigations, or both. Even in shorter longitudinal studies, it has been a challenge to maintain the motivation of the participants unless the study is conducted in special circumstances such as summer camp like the study environment in the innovative study by Moreno et al. (2011). Thus, the current choice of 
having a longitudinal study on children who chose their music training based on their own and their family's initiative, gives solid evidence about the development of cognitive functions of music-oriented and control children obtained in an ecologically valid context.

\section{SUMMARY AND CONCLUSIONS}

In this study, we investigated the maturation of working memory in musically trained and nontrained children and adolescents. We found different patterns of development for different subcomponents of working memory in the trained and nontrained participants. Musically trained individuals had better performance in tests tapping working memory maintenance, but not updating, than musically nontrained individuals. However, the difference lessened over time, as nontrained participants attained a similar level of performance as trained participants. Our results extend previous findings of a musician advantage in tests for working memory by specifying which subcomponents of working memory may be most affected, and by clarifying the trajectory of enhancement from childhood into adolescence.

\section{DATA AVAILABILITY STATEMENT}

The datasets for this manuscript are not publicly available because the consent form signed by participants did not include

\section{REFERENCES}

Aben, B., Stapert, S., and Blokland, A. (2012). About the distinction between working memory and short-term memory. Front. Psychol. 3:301. doi: 10.3389/fpsyg.2012.00301

Arbuthnott, K., and Frank, J. (2000). Trail making test, part B as a measure of executive control: validation using a set-switching paradigm. J. Clin. Exp. Neuropsychol. 22, 518-528. doi: 10.1076/1380-3395(200008)22:4;1-0;FT518

Baddeley, A. D., and Hitch, G. (1974). "Working memory," in Psychology of Learning and Motivation (Vol. 8), ed. G. H. Bower (New York, NY: Academic Press), 47-89.

Barbaroux, M., Dittinger, E., and Besson, M. (2019). Music training with Démos program positively influences cognitive functions in children from low socioeconomic backgrounds. PLoS One 14:e0216874. doi: 10.1371/journal.pone. 0216874

Bates, D. (2005). Fitting linear mixed models in R. R News 5, 27-30.

Bates, D., Sarkar, D., Bates, M. D., and Matrix, L. (2007). The lme4 package. $R$ Package Version 2:74.

Bergman Nutley, S., Darki, F., and Klingberg, T. (2014). Music practice is associated with development of working memory during childhood and adolescence. Front. Hum. Neurosci. 7:926. doi: 10.3389/fnhum.2013. 00926

Bermudez, P., Lerch, J. P., Evans, A. C., and Zatorre, R. J. (2008). Neuroanatomical correlates of musicianship as revealed by cortical thickness and voxel-based morphometry. Cereb. Cortex 19, 1583-1596. doi: 10.1093/cercor/bhn196

Berry, A. S., Zanto, T. P., Rutman, A. M., Clapp, W. C., and Gazzaley, A. (2009). Practice-related improvement in working memory is modulated by changes in processing external interference. J. Neurophysiol. 102, 1779-1789. doi: 10.1152/jn.00179.2009

Best, J. R., and Miller, P. H. (2010). A developmental perspective on executive function. Child Dev. 81, 1641-1660. doi: 10.1111/j.1467-8624.2010.01499.x

Bialystok, E., and Depape, A. M. (2009). Musical expertise, bilingualism, and executive functioning. J. Exp. Psychol. Hum. Percept. Perform. 35, 565-574. doi: $10.1037 / \mathrm{a} 0012735$ permission for distribution of data outside the research group. Requests to access the datasets should be directed to Katri Saarikivi, katri.saarikivi@helsinki.fi.

\section{ETHICS STATEMENT}

The studies involving human participants were reviewed and approved by The University of Helsinki Ethical Review Board in the Humanities and Social and Behavioural Sciences. Written informed consent to participate in this study was provided by the participants' legal guardian/next of kin.

\section{AUTHOR CONTRIBUTIONS}

KS and VP participated in planning the experiment, conducting the measurements, analyzing the data and writing the manuscript. MT and MH were responsible for establishing the longitudinal study, planning the experiments and measurement paradigms included, and reviewing and writing the manuscript.

\section{FUNDING}

This research has been funded by The Jenny and Antti Wihuri Foundation, The Finnish Cultural Foundation and The Academy of Finland.

Blackwell, K. A., Cepeda, N. J., and Munakata, Y. (2009). When simple things are meaningful: working memory strength predicts children's cognitive flexibility. J. Exp. Child Psychol. 103, 241-249. doi: 10.1016/j.jecp.2009.01.002

Bugos, J., and Mostafa, W. (2011). Musical training enhances information processing speed. Bull. Council Res. Music Educ. 187, 7-18.

Bunge, S. A., and Wright, S. B. (2007). Neurodevelopmental changes in working memory and cognitive control. Curr. Opin. Neurobiol. 17, 243-250. doi: 10.1016/j.conb.2007.02.005

Carretti, B., Borella, E., and De Beni, R. (2007). Does strategic memory training improve the working memory performance of younger and older adults? Exp. Psychol. 54, 311-320. doi: 10.1027/1618-3169.54.4.311

Casey, B. J., Giedd, J. N., and Thomas, K. M. (2000). Structural and functional brain development and its relation to cognitive development. Biol. Psychol. 54, 241-257. doi: 10.1016/s0301-0511(00)00058-2

Cepeda, N. J., Kramer, A. F., and Gonzalez de Sather, J. C. M. (2001). Changes in executive control across the life span: examination of task-switching performance. Dev. Psychol. 37, 715-730. doi: 10.1037/0012-1649.37. 5.715

Chan, A. S., Ho, Y. C., and Cheung, M. C. (1998). Music training improves verbal memory. Nature 396:128. doi: 10.1038/24075

Cheung, M. C., Chan, A. S., Liu, Y., Law, D., and Wong, C. W. (2017). Music training is associated with cortical synchronization reflected in EEG coherence during verbal memory encoding. PLoS One 12:e0174906. doi: 10.1371/journal. pone.0174906

Cowan, N. (1988). Evolving conceptions of memory storage, selective attention, and their mutual constraints within the human information-processing system. Psychol. Bull. 104, 163-191. doi: 10.1037/0033-2909.104.2.163

Cowan, N. (1999). "An embedded-processes model of working memory," in Models of Working Memory: Mechanisms of Active Maintenance and Executive Control (Vol. 20), eds A. Miyake and P. Shah (Cambridge, MA: Cambridge University Press), 506.

Cowan, N. (2008). What are the differences between long-term, short-term, and working memory? Prog. Brain Res. 169, 323-338. doi: 10.1016/s00796123(07)00020-9 
De Luca, C. R., Wood, S. J., Anderson, V., Buchanan, J.-A., Proffitt, T. M., Mahony, K., et al. (2003). Normative data from the CANTAB. I: development of executive function over the lifespan. J. Clin. Exp. Neuropsychol. 25, 242-254. doi: 10.1076/jcen.25.2.242.13639

Degé, F., Kubicek, C., and Schwarzer, G. (2011). Music lessons and intelligence: a relation mediated by executive functions. Music Percept. 29, 195-201. doi: 10.1525/mp.2011.29.2.195

Demetriou, A., Spanoudis, G., Shayer, M., Van der Ven, S., Brydges, C. R., Kroesbergen, E., et al. (2014). Relations between speed, working memory, and intelligence from preschool to adulthood: structural equation modeling of 14 studies. Intelligence 46, 107-121. doi: 10.1016/j.intell.2014.05.013

D’Esposito, M., Detre, J. A., Alsop, D. C., Shin, R. K., Atlas, S., and Grossman, M. (1995). The neural basis of the central executive system of working memory. Nature 378, 279-281. doi: 10.1038/378279a0

Diamond, A. (2013). Executive functions. Annu. Rev. Psychol. 64, 135-168. doi: 10.1146/annurev-psych-113011-143750

Ding, Y., Gray, K., Forrence, A., Wang, X., and Huang, J. (2018). A behavioral study on tonal working memory in musicians and non-musicians. PLoS One 13:e0201765. doi: 10.1371/journal.pone.0201765

Ellis, R. J., Molander, P., Rönnberg, J., Lyxell, B., Andersson, G., and Lunner, T. (2016). Predicting speech-in-noise recognition from performance on the trail making test: results from a large-scale internet study. Ear Hear. 37, 73-79. doi: 10.1097/aud.0000000000000218

Eriksson, J., Vogel, E. K., Lansner, A., Bergström, F., and Nyberg, L. (2015). Neurocognitive architecture of working memory. Neuron 88, 33-46. doi: 10.1016/j.neuron.2015.09.020

Fellows, R. P., Dahmen, J., Cook, D., and Schmitter-Edgecombe, M. (2017). Multicomponent analysis of a digital Trail Making Test. Clin. Neuropsychol. 31, 154-167. doi: 10.1080/13854046.2016.1238510

Fujioka, T., Ross, B., Kakigi, R., Pantev, C., and Trainor, L. J. (2006). One year of musical training affects development of auditory cortical-evoked fields in young children. Brain 129, 2593-2608. doi: 10.1093/brain/awl247

Fuster, J. M. (2002). Frontal lobe and cognitive development. J. Neurocytol. 31, 373-385. doi: 10.1023/a:1024190429920

Gaser, C., and Schlaug, G. (2003). Brain structures differ between musicians and non-musicians. J. Neurosci. 23, 9240-9245. doi: 10.1523/JNEUROSCI.23-2709240.2003

Gathercole, S. E., Pickering, S. J., Ambridge, B., and Wearing, H. (2004). The structure of working memory from 4 to 15 years of age. Dev. Psychol. 40, 177-190. doi: 10.1037/0012-1649.40.2.177

Gazzaley, A., and Nobre, A. C. (2012). Top-down modulation: bridging selective attention and working memory. Trends Cogn. Sci. 16, 129-135. doi: 10.1016/j. tics.2011.11.014

George, E. M., and Coch, D. (2011). Music training and working memory: an ERP study. Neuropsychologia 49, 1083-1094. doi: 10.1016/j.neuropsychologia.2011. 02.001

Gerton, B. K., Brown, T. T., Meyer-Lindenberg, A., Kohn, P., Holt, J. L., Olsen, R. K., et al. (2004). Shared and distinct neurophysiological components of the digits forward and backward tasks as revealed by functional neuroimaging. Neuropsychologia 42, 1781-1787. doi: 10.1016/j. neuropsychologia.2004.04.023

Getzmann, S., Wascher, E., and Schneider, D. (2018). The role of inhibition for working memory processes: ERP evidence from a short-term storage task. Psychophysiology 55:e13026. doi: 10.1111/psyp.13026

Gray, S., Green, S., Alt, M., Hogan, T., Kuo, T., Brinkley, S., et al. (2017). The structure of working memory in young children and its relation to intelligence. J. Mem. Lang. 92, 183-201. doi: 10.1016/j.jml.2016.06.004

Guo, X., Ohsawa, C., Suzuki, A., and Sekiyama, K. (2018). Improved digit span in children after a 6-week intervention of playing a musical instrument: an exploratory randomized controlled trial. Front. Psychol. 8:2303. doi: 10.3389/fpsyg.2017.02303

Hagenaars, S. P., Cox, S. R., Hill, W. D., Davies, G., Liewald, D. C., Harris, S. E., et al. (2018). Genetic contributions to trail making test performance in UK Biobank. Mol. Psychiatry 23, 1575-1583. doi: 10.1038/mp. 2017.189

Hansen, M., Wallentin, M., and Vuust, P. (2013). Working memory and musical competence of musicians and non-musicians. Psychol. Music 41, 779-793. doi: $10.1177 / 0305735612452186$
Heinzel, S., Lorenz, R. C., Pelz, P., Heinz, A., Walter, H., Kathmann, N., et al. (2016). Neural correlates of training and transfer effects in working memory in older adults. Neuroimage 134, 236-249. doi: 10.1016/j.neuroimage.2016. 03.068

Ho, Y. C., Cheung, M. C., and Chan, A. S. (2003). Music training improves verbal but not visual memory: cross-sectional and longitudinal explorations in children. Neuropsychology 17, 439-450. doi: 10.1037/0894-4105.17. 3.439

Huizinga, M., Dolan, C. V., and van der Molen, M. W. (2006). Age-related change in executive function: developmental trends and a latent variable analysis. Neuropsychologia 44, 2017-2036. doi: 10.1016/j.neuropsychologia.2006. 01.010

Huizinga, M., and van der Molen, M. W. (2011). Task switching and shifting between stopping and going: developmental change in between-trial control adjustments. J. Exp. Child Psychol. 108, 484-503. doi: 10.1016/j.jecp.2010. 10.004

James, C. E., Oechslin, M. S., Van De Ville, D., Hauert, C. A., Descloux, C., and Lazeyras, F. (2014). Musical training intensity yields opposite effects on grey matter density in cognitive versus sensorimotor networks. Brain Struct. Funct. 219, 353-366. doi: 10.1007/s00429-013-0504-z

Jonides, J., Smith, E. E., Marshuetz, C., Koeppe, R. A., and Reuter-Lorenz, P. A. (1998). Inhibition in verbal working memory revealed by brain activation. Proc. Natl. Acad. Sci. U S A 95, 8410-8413. doi: 10.1073/pnas.95.14.8410

Jurado, M. B., and Rosselli, M. (2007). The elusive nature of executive functions: a review of our current understanding. Neuropsychol. Rev. 17, 213-233. doi: 10.1007/s11065-007-9040-z

Kail, R. V. (2007). Longitudinal evidence that increases in processing speed and working memory enhance children's reasoning. Psychol. Sci. 18, 312-313. doi: 10.1111/j.1467-9280.2007.01895.x

Kane, M. J., Hambrick, D. Z., and Conway, A. R. (2005). Working memory capacity and fluid intelligence are strongly related constructs: comment on Ackerman, Beier, and Boyle (2005). Psychol. Bull. 131, 66-71. doi: 10.1037/0033-2909.131.1.66

Kharitonova, M., Martin, R. E., Gabrieli, J. D. E., and Sheridan, M. A. (2013). Cortical gray-matter thinning is associated with age-related improvements on executive function tasks. Dev. Cogn. Neurosci. 6, 61-71. doi: 10.1016/j.dcn.2013. 07.002

Kwon, H., Reiss, A. L., and Menon, V. (2002). Neural basis of protracted developmental changes in visuo-spatial working memory. Proc. Natl. Acad. Sci. U S A 99, 13336-13341. doi: 10.1073/pnas.162486399

Lee, Y. S., Lu, M. J., and Ko, H. P. (2007). Effects of skill training on working memory capacity. Learn. Instr. 17, 336-344. doi: 10.1016/j.learninstruc.2007. 02.010

Lehto, J. E., Juujärvi, P., Kooistra, L., and Pulkkinen, L. (2003). Dimensions of executive functioning: evidence from children. Br. J. Dev. Psychol. 21, 59-80. doi: 10.1348/026151003321164627

Linares, R., Borella, E., Lechuga, M. T., Carretti, B., and Pelegrina, S. (2018). Training working memory updating in young adults. Psychol. Res. 82, 535-548. doi: 10.1007/s00426-017-0843-0

Linares, R., Borella, E., Lechuga, M. T., Carretti, B., and Pelegrina, S. (2019). Nearest transfer effects of working memory training: A comparison of two programs focused on working memory updating. PLoS One 14:e0211321. doi: 10.1371/journal.pone.0211321

Linden, D. E. (2007). The working memory networks of the human brain. Neuroscientist 13, 257-267. doi: 10.1177/1073858406298480

Llinàs-Reglà, J., Vilalta-Franch, J., López-Pousa, S., Calvó-Perxas, L., Torrents Rodas, D., and Garre-Olmo, J. (2017). The trail making test: association with other neuropsychological measures and normative values for adults aged 55 years and older from a Spanish-Speaking Population-based sample. Assessment 24, 183-196. doi: 10.1177/1073191115602552

Luna, B., Garver, K. E., Urban, T. A., Lazar, N. A., and Sweeney, J. A. (2004). Maturation of cognitive processes from late childhood to adulthood. Child Dev. 75, 1357-1372. doi: 10.1111/j.1467-8624.2004.00745.x

Manan, H. A., Franz, E. A., Yusoff, A. N., and Mukari, S. Z. M. S. (2014). Agerelated brain activation during forward and backward verbal memory tasks. Neurol. Psychiatry Brain Res. 20, 76-86. doi: 10.1016/j.npbr.2014.08.001

Melby-Lervåg, M., and Hulme, C. (2013). Is working memory training effective? A meta-analytic review. Dev. Psychol. 49, 270-291. doi: 10.1037/a0028228 
Miller, E. K., and Cohen, J. D. (2001). An integrative theory of prefrontal cortex function. Annu. Rev. Neurosci. 24, 167-202. doi: 10.1146/annurev.neuro. 24.1.167

Miyake, A., and Friedman, N. P. (2012). The nature and organization of individual differences in executive functions four general conclusions. Curr. Dir. Psychol. Sci. 21, 8-14. doi: 10.1177/0963721411429458

Miyake, A., Friedman, N. P., Emerson, M. J., Witzki, A. H., Howerter, A., and Wager, T. D. (2000). The unity and diversity of executive functions and their contributions to complex "frontal lobe" tasks: a latent variable analysis. Cogn. Psychol. 41, 49-100. doi: 10.1006/cogp.1999.0734

Moreno, S., Bialystok, E., Barac, R., Schellenberg, E. G., Cepeda, N. J., and Chau, T. (2011). Short-term music training enhances verbal intelligence and executive function. Psychol. Sci. 22, 1425-1433. doi: 10.1177/0956797611 416999

Morrison, A. B., and Chein, J. M. (2011). Does working memory training work? The promise and challenges of enhancing cognition by training working memory. Psychon. Bull. Rev. 18, 46-60. doi: 10.3758/s13423-010-0034-0

Nee, D. E., Brown, J. W., Askren, M. K., Berman, M. G., Demiralp, E., Krawitz, A., et al. (2012). A meta-analysis of executive components of working memory. Cereb. Cortex 23, 264-282. doi: 10.1093/cercor/bhs007

Owen, A. M., McMillan, K. M., Laird, A. R., and Bullmore, E. (2005). N-back working memory paradigm: a meta-analysis of normative functional neuroimaging studies. Hum. Brain Mapp. 25, 46-59. doi: 10.1002/hbm.20131

Pallesen, K. J., Brattico, E., Bailey, C. J., Korvenoja, A., Koivisto, J., Gjedde, A., et al. (2010). Cognitive control in auditory working memory is enhanced in musicians. PLoS One 5:e11120. doi: 10.1371/journal.pone.0011120

Postle, B. R., Berger, J. S., and D'Esposito, M. (1999). Functional neuroanatomical double dissociation of mnemonic and executive control processes contributing to working memory performance. Proc. Natl. Acad. Sci. U S A 96, 12959-12964. doi: 10.1073/pnas.96.22.12959

Poutiainen, E., Kalska, H., Laasonen, M., Närhi, V., and Räsänen, P. (2010). Trail Making Testi. Helsinki: Psykologien Kustannus Oy.

Putkinen, V., Tervaniemi, M., Saarikivi, K., Ojala, P., and Huotilainen, M. (2014). Enhanced development of auditory change detection in musically trained school-aged children: a longitudinal event-related potential study. Dev. Sci. 17, 282-297. doi: 10.1111/desc.12109

Redick, T. S., and Lindsey, D. R. (2013). Complex span and n-back measures of working memory: a meta-analysis. Psychon. Bull. Rev. 20, 1102-1113. doi: 10.3758/s13423-013-0453-9

Roden, I., Grube, D., Bongard, S., and Kreutz, G. (2014). Does music training enhance working memory performance? Findings from a quasi-experimental longitudinal study. Psychol. Music 42, 284-298. doi: $10.1177 / 0305735612471239$

Rottschy, C., Langner, R., Dogan, I., Reetz, K., Laird, A. R., Schulz, J. B., et al. (2012). Modelling neural correlates of working memory: a coordinatebased meta-analysis. Neuroimage 60, 830-846. doi: 10.1016/j.neuroimage.2011. 11.050

Saarikivi, K., Putkinen, V., Tervaniemi, M., and Huotilainen, M. (2016). Cognitive flexibility modulates maturation and music-training-related changes in neural sound discrimination. Eur. J. Neurosci. 44, 1815-1825. doi: 10.1111/ejn. 13176

Sala, G., and Gobet, F. (2017a). When the music's over. Does music skill transfer to children's and young adolescents' cognitive and academic skills? A metaanalysis. Educ. Res. Rev. 20, 55-67. doi: 10.1016/j.edurev.2016.11.005

Sala, G., and Gobet, F. (2017b). Working memory training in typically developing children: a meta-analysis of the available evidence. Dev. Psychol. 53, 671-685. doi: $10.1037 /$ dev0000265

Salthouse, T. A. (2014). Relations between running memory and fluid intelligence. Intelligence 43, 1-7. doi: 10.1016/j.intell.2013.12.002

Sánchez-Cubillo, I., Periáñez, J. A., Adrover-Roig, D., Rodríguez-Sánchez, J. M., Ríos-Lago, M., Tirapu, J. E. E. A., et al. (2009). Construct validity of the trail making test: role of task-switching, working memory, inhibition/interference control and visuomotor abilities. J. Int. Neuropsychol. Soc. 15, 438-450. doi: 10. 1017/S1355617709090626

Satterthwaite, T. D., Wolf, D. H., Erus, G., Ruparel, K., Elliott, M. A., Gennatas, E. D., et al. (2013). Functional maturation of the executive system during adolescence. J. Neurosci. 33, 16249-16261. doi: 10.1523/JNEUROSCI. 2345-13.2013
Schellenberg, E. G. (2004). Music lessons enhance IQ. Psychol. Sci. 15, 511-514. doi: 10.1111/j.0956-7976.2004.00711.x

Schellenberg, E. G. (2006). Long-term positive associations between music lessons and IQ. J. Educ. Psychol. 98, 457-468. doi: 10.1037/0022-0663.98.2.457

Schellenberg, E. G. (2011). Examining the association between music lessons and intelligence. Br. J. Psychol. 102, 283-302. doi: 10.1111/j.2044-8295.2010. 02000.x

Schellenberg, E. G. (2015). Music training and speech perception: a gene-environment interaction. Ann. NY Acad. Sci. 1337, 170-177. doi: $10.1111 /$ nyas. 12627

Schleepen, T. M., and Jonkman, L. M. (2009). The development of non-spatial working memory capacity during childhood and adolescence and the role of interference control: an N-Back task study. Dev. Neuropsychol. 35, 37-56. doi: $10.1080 / 87565640903325733$

Schulze, K., Müller, K., and Koelsch, S. (2011). Neural correlates of strategy use during auditory working memory in musicians and non-musicians. Eur. J. Neurosci. 33, 189-196. doi: 10.1111/j.1460-9568.2010. 07470.x

Siffredi, V., Barrouillet, P., Spencer-Smith, M., Vaessen, M., Anderson, V., and Vuilleumier, P. (2017). Examining distinct working memory processes in children and adolescents using fMRI: results and validation of a modified Brown-Peterson paradigm. PLoS One 12:e0179959. doi: 10.1371/journal.pone. 0179959

Sreenivasan, K. K., and Jha, A. P. (2007). Selective attention supports working memory maintenance by modulating perceptual processing of distractors. J. Cogn. Neurosci. 19, 32-41. doi: 10.1162/jocn.2007.19.1.32

Stoodley, C. J., Valera, E. M., and Schmahmann, J. D. (2012). Functional topography of the cerebellum for motor and cognitive tasks: an fMRI study. Neuroimage 59, 1560-1570. doi: 10.1016/j.neuroimage.2011. 08.065

Strait, D. L., and Kraus, N. (2011). Can you hear me now? Musical training shapes functional brain networks for selective auditory attention and hearing speech in noise. Front. Psychol. 2:113. doi: 10.3389/fpsyg.2011.00113

Strait, D. L., Slater, J., O'Connell, S., and Kraus, N. (2015). Music training relates to the development of neural mechanisms of selective auditory attention. Dev. Cogn. Neurosci. 12, 94-104. doi: 10.1016/j.dcn.2015.01.001

Strong, J. V., and Mast, B. T. (2019). The cognitive functioning of older adult instrumental musicians and non-musicians. Neuropsychol. Dev. Cogn. B Aging Neuropsychol. Cogn. 26, 367-386. doi: 10.1080/13825585.2018. 1448356

Stuss, D. T., and Alexander, M. P. (2000). Executive functions and the frontal lobes: a conceptual view. Psychol. Res. 63, 289-298. doi: 10.1007/s004269900007

Talamini, F., Altoè, G., Carretti, B., and Grassi, M. (2017). Musicians have better memory than nonmusicians: a meta-analysis. PLoS One 12:e0186773. doi: 10.1371/journal.pone.0186773

Talamini, F., Carretti, B., and Grassi, M. (2016). The working memory of musicians and nonmusicians. Music Percept. 34, 183-191. doi: 10.1525/mp. 2016.34.2.183

Taylor, S. J., Barker, L. A., Heavey, L., and McHale, S. (2013). The typical developmental trajectory of social and executive functions in late adolescence and early adulthood. Dev. Psychol. 49, 1253-1265. doi: 10.1037/a0029871

Taylor, S. J., Barker, L. A., Heavey, L., and McHale, S. (2015). The longitudinal development of social and executive functions in late adolescence and early adulthood. Front. Behav. Neurosci. 9:252. doi: 10.3389/fnbeh.2015.00252

Tervaniemi, M., Tao, S., and Huotilainen, M. (2018). Promises of music in education? Front. Educ. 3:74. doi: 10.3389/feduc.2018.00074

Unsworth, N., and Engle, R. W. (2007). The nature of individual differences in working memory capacity: active maintenance in primary memory and controlled search from secondary memory. Psychol. Rev. 114, 104-132. doi: 10.1037/0033-295x.114.1.104

Varjacic, A., Mantini, D., Demeyere, N., and Gillebert, C. R. (2018). Neural signatures of Trail Making Test performance: evidence from lesion-mapping and neuroimaging studies. Neuropsychologia 115, 78-87. doi: 10.1016/j. neuropsychologia.2018.03.031

Virtala, P., Huotilainen, M., Partanen, E., and Tervaniemi, M. (2014). Musicianship facilitates the processing of Western music chords-an ERP and behavioral study. Neuropsychologia 61, 247-258. doi: 10.1016/j. neuropsychologia.2014.06.028 
Vuontela, V., Steenari, M. R., Carlson, S., Koivisto, J., Fjällberg, M., and Aronen, E. T. (2003). Audiospatial and visuospatial working memory in 6-13 year old school children. Learn. Mem. 10, 74-81. doi: 10.1101/lm.53503

Wang, J., Tian, J., Hao, R., Tian, L., and Liu, Q. (2018). Transcranial direct current stimulation over the right DLPFC selectively modulates subprocesses in working memory. PeerJ 6:e4906. doi: 10.7717/peerj.4906

Wechsler, D. (2010). Wechsler Intelligence Scale for Children IV. Finland: Psykologien Kustannus.

Wilhelm, O., Hildebrandt, A. H., and Oberauer, K. (2013). What is working memory capacity, and how can we measure it? Front. Psychol. 4:433. doi: 10.3389/fpsyg.2013.00433

Yang, Z., Jutagir, D. R., Koyama, M. S., Craddock, R. C., Yan, C. G., Shehzad, Z., et al. (2015). Intrinsic brain indices of verbal working memory capacity in children and adolescents. Dev. Cogn. Neurosci. 15, 67-82. doi: 10.1016/j.dcn. 2015.07.007

Zanto, T. P., and Gazzaley, A. (2009). Neural suppression of irrelevant information underlies optimal working memory performance. J. Neurosci. 29, 3059-3066. doi: 10.1523/jneurosci.4621-08.2009
Zelazo, P. D., Craik, F. I. M., and Booth, L. (2004). Executive function across the life span. Acta Psychol. 115, 167-183. doi: 10.1016/j.actpsy.2003. 12.005

Zuk, J., Benjamin, C., Kenyon, A., and Gaab, N. (2014). Behavioral and neural correlates of executive functioning in musicians and non-musicians. PLoS One 9:e99868. doi: 10.1371/journal.pone.0099868

Conflict of Interest: The authors declare that the research was conducted in the absence of any commercial or financial relationships that could be construed as a potential conflict of interest.

Copyright (c) 2019 Saarikivi, Huotilainen, Tervaniemi and Putkinen. This is an open-access article distributed under the terms of the Creative Commons Attribution License (CC BY). The use, distribution or reproduction in other forums is permitted, provided the original author(s) and the copyright owner(s) are credited and that the original publication in this journal is cited, in accordance with accepted academic practice. No use, distribution or reproduction is permitted which does not comply with these terms. 